\title{
A DYNAMO MODEL FOR THE LARGE SCALE FIELDS
}

\author{
I. K. CSADA \\ Konkoly Observatory, Budapest, Hungary
}

\begin{abstract}
The expansion of the magnetic field in a series of force-free constituents for the internal field and that of 'semi' force-free constituents for the external field results in the skew symmetry of the coupling tensor of a magnetohydrodynamic dynamo. The periodic solution which exists in this case is deduced by the method of eigenvalues. Based on the numerical results a dynamo model is proposed to draw up the characteristic features of the magnetographic observations carried along the central meridian and at the polar areas.
\end{abstract}

The coupling tensor $\alpha_{s n}$ in the eigenvalue theory of the magnetohydrodynamic dynamo is transformed to skew-symmetric form. In this case the solutions to the dynamo equation give a magnetic field with periodical variation superimposed on decays. The velocity is steady.

On applying Gauss's integral theorem, the tensor $\alpha_{s n}$ can be written in the form

$$
\alpha_{s n}=-\frac{\int \mathbf{v}\left(\operatorname{curl} \mathbf{H}_{s} \times \mathbf{H}_{n}\right) \mathrm{d} \tau}{\int H_{s}^{2} \mathrm{~d} \tau} .
$$

The surface integral involved in the complete expression goes here to zero owing to the boundary conditions imposed on the velocity and magnetic field.

Two methods are available for the transformation of $\alpha$ to skew-symmetric form.

(1) Let $\mathbf{H}_{n}$ be a force-free vector satisfying the equation

$$
\operatorname{curl} \mathbf{H}_{n} \times \mathbf{H}_{n}=0 \text {. }
$$

(The magnetic field is not force-free since it is defined as

$$
\mathbf{H}=\sum_{n=0} B_{n}(t) \mathbf{H}_{n}(r, \theta, \phi)
$$

and the sum of force-free vectors is not force-free).

Applying the dynamo to the internal field of the Sun, $\mathbf{H}_{n}$ is governed by the equation

$$
\Delta \mathbf{H}_{n}+\frac{k_{n}^{2}}{\kappa} \mathbf{H}_{n}=0
$$

and the coupling tensor has the form

$$
\alpha_{s n}=-\frac{k_{n} \int \mathbf{v}\left(\mathbf{H}_{s} \times \mathbf{H}_{n}\right) \mathrm{d} \tau}{\int H_{n}^{2} \mathrm{~d} \tau} .
$$


(2) In the other method one uses the assumption that only the $r$ and $\theta$ components of $\mathbf{H}_{n}$ have force-free character and the velocity has only a $\phi$ component, then

$$
\alpha_{s n}=-\frac{k_{s} \int v_{\phi}\left|\begin{array}{ll}
H_{s r} & H_{\delta \theta} \\
H_{n r} & H_{n \theta}
\end{array}\right| \mathrm{d} \tau}{\int H_{s}^{2} \mathrm{~d} \tau}
$$

which can be easily transformed to skew-symmetric form.

Applying this formulation to the external field, $\mathbf{H}_{n}$ is governed by the equation

$$
\Delta \mathbf{H}_{n}-\frac{k_{n}^{2}}{\kappa} \mathbf{H}_{n}=0 .
$$

In the next step the infinite tensor $\alpha_{s n}$ is reduced to a finite tensor by the modification of the velocity and thus the infinite determinant of $\alpha_{s n}$ splits into the double product

$$
\left|\alpha_{s n}-i \Omega \delta_{s n}\right|=D_{1} D_{2}=0,
$$

where $D_{1}$ is a finite and $D_{2}$ is an infinite determinant. By putting $D_{1}=0$, no higher terms than those present at the start of the dynamo will be generated.

Two models were computed in terms of the present dynamo. Both have the same internal field but in the first case the external field is assumed to be random without any average contribution to the field fluctuations while in the second no random fluctuations are supposed to occur in the external field deduced from (6) and (7).

The numerical and analytical results obtained in these models seem to account for the interpretation of the following phenomena in solar physics.

(i) The cyclical variation of the general field. In a general expansion of the theory the cyclical variation is presented by the superposition of a large number of periodic terms arising in $B_{n}(t)$. In the present solution the analysis is performed up to the first four terms of (3) and thus two periods were obtained. The periods evaluated with the use of the second model are about ten times shorter than the corresponding periods deduced in the first model, i.e.

$$
\begin{array}{lll}
P_{1}=27.36 \mathrm{yr} & P_{2}=137.45 \mathrm{yr} & \text { first model } \\
P_{1}=1.60 \mathrm{yr} & P_{2}=95.33 \mathrm{yr} & \text { second model }
\end{array}
$$

It seems reasonable to describe the cyclical variations of the solar activity by coupling of the two models. Thus, the second model applies to the minimum since its time scale is short and negligible magnetic disturbances are observed in the photosphere, while the first model, having a longer time scale during which practically no general field is present in terms of the averages of the magnetic fluctuations, holds for the maximum.

(ii) The average surface distribution and the variation of weak photospheric fields. These phenomena are accounted for in terms of the second model. The field component of the line of sight is calculated for the central meridian as a function of time (Figure 1). These results are intended to explain the magnetogram observations averaged over rotation periods. 
(iii) The field reversal observed at the poles is deduced by the first model (Figure 2). No simple variation of the polar field is obtained. As an approximate law of variation one can calculate with the superposition of two periods. The longer period has a larger amplitude at one of the poles while at the other the amplitude of the shorter period is longer.

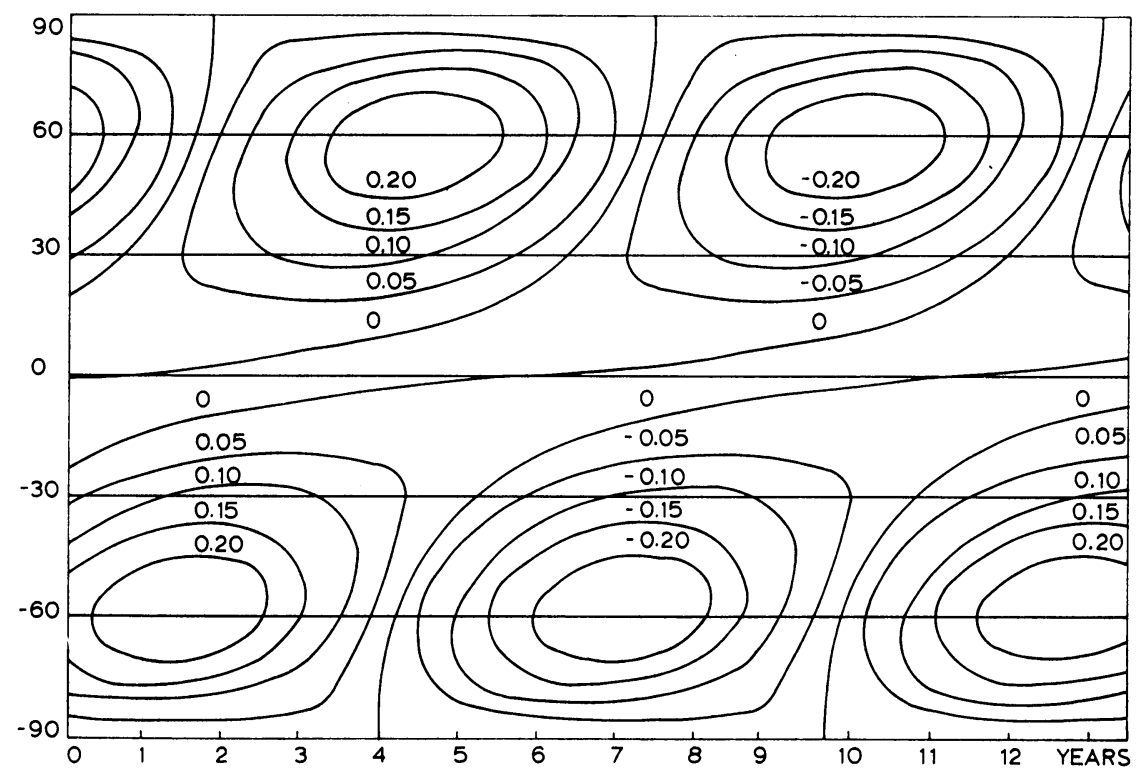

Fig. 1. Characteristic variation of the magnetic field (in arbitrary units) as it should be observed along the central meridian and averaged over rotation periods during the solar cycles. The results are based on the present dynamo model. The solar latitude is plotted on the vertical axis, the time on the horizontal axis.

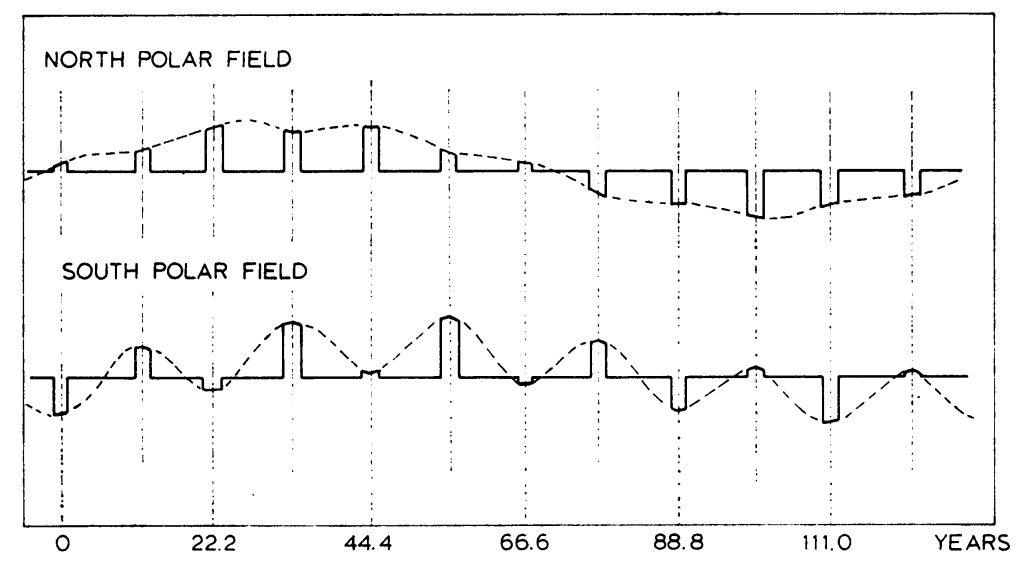

Fig. 2. The variations of the polar fields during the solar cycles as they are obtained in the present theory. The field strengths are measured in arbitrary units. 